\title{
Existence of Positive Solutions for Multi-Point Boundary Value Problems on Infinite Intervals in Banach Spaces
}

\author{
Zhaocai Hao and Liang Ma \\ School of Mathematical Sciences, Qufu Normal University, Shandong, Qufu 273165, China \\ Correspondence should be addressed to Zhaocai Hao, zchjal@163.com \\ Received 18 July 2012; Accepted 12 September 2012 \\ Academic Editor: Xinguang Zhang
}

Copyright (C) 2012 Z. Hao and L. Ma. This is an open access article distributed under the Creative Commons Attribution License, which permits unrestricted use, distribution, and reproduction in any medium, provided the original work is properly cited.

We investigate the positive solutions of a class of second-order nonlinear singular differential equations with multi-point boundary value conditions on an infinite interval in Banach spaces. The tools we used are the cone theory and Mönch fixed point theorem and a monotone iterative technique. An example is also given to demonstrate the applications of our results, which include and extend some existing results.

\section{Introduction}

Let $E$ be a real Banach space and let $P$ be a cone in $E$. $P$ is said to be normal if there exists a positive constant $N$ such that $\theta \leq x \leq y$ implies $\|x\| \leq N\|y\|$. $P$ is said to be regular (fully regular) if $u_{1} \leq u_{2} \leq \cdots \leq u_{n} \leq y \cdots$, $\sup _{n}\left\|u_{n}\right\|<\infty$ implies that their exists $x \in E$ such that $\lim _{n \rightarrow \infty}\left\|u_{n}-x\right\|=0$. Let $P_{+}=P \backslash\{\theta\}$. In what follows, we always assume that $x_{0}^{*} \in P_{+}$. Let $P_{0 \lambda}=\left\{u \in P: u \geq \lambda x_{0}^{*}\right\}(\lambda>0)$. Obviously, $P_{0 \lambda} \subset P_{+}$for any $\lambda>0$. When $\lambda=1$, we write $P_{0}=P_{01}$, that is, $P_{0}=\left\{u \in P: u \geq x_{0}^{*}\right\}$.

In this paper, we will consider the following boundary value problems (BVPs) for multipoint singular differential equations of mixed type on an unbounded domain in a real Banach space $(E,\|\cdot\|)$

$$
\begin{aligned}
& u^{\prime \prime}(t)+f\left(t, u(t), u^{\prime}(t),(T u(t)),(S u(t))\right)=\theta, \quad t \in J_{+}, \\
& a u(0)-b u^{\prime}(0)-\sum_{i=1}^{n} k_{i} u\left(\xi_{i}\right)=\theta, \quad \lim _{t \rightarrow+\infty} u^{\prime}(t)=y_{\infty},
\end{aligned}
$$


where $J=[0,+\infty), J_{+}=(0,+\infty), a>0, b \geq 0, k_{i} \geq 0,0<\xi_{1}<\xi_{2}<\cdots<\xi_{n}<+\infty, f \in$ $C\left[J_{+} \times P_{0 \lambda} \times P_{0 \lambda} \times P \times P, P\right], \curlywedge>0, y_{\infty} \geq x_{0}^{*}$,

$$
(T u)(t)=\int_{0}^{t} K(t, s) u(s) d s, \quad(S u)(t)=\int_{0}^{\infty} H(t, s) u(s) d s
$$

$K \in C[D, J], D=\{(t, s) \in J \times J: t \geq s\}, H \in C[J \times J, J]$. Here the nonlinear term $f$ may be singular at $t=0$ and $x_{0}, x_{1}, x_{2}, x_{3}=\theta$. By singularity, we mean that $\left\|f\left(t, x_{0}, x_{1}, x_{2}, x_{3}\right)\right\| \rightarrow \infty$ as $t \rightarrow 0^{+}$or $x_{i} \rightarrow \theta^{+}(i=0,1,2,3)$.

Second-order boundary value problems (BVPs) on infinite intervals, arising from the study of radially symmetric solutions of nonlinear elliptic equation and models of gas pressure in a semi-infinite porous medium, have received much attention. We can see papers $[1,2]$ and the references therein. In a recent paper, Liu [3] investigated the existence of solutions of the following second-order two-point boundary value problems on the half-line:

$$
\begin{gathered}
x^{\prime \prime}(t)+f(t, x(t))=0, \quad t \in(0,+\infty), \\
x(0)=0, \quad x^{\prime}(\infty)=y_{\infty}>0 .
\end{gathered}
$$

Lian and Ge [4] studied the solvability of the three-point BVP

$$
\begin{gathered}
u^{\prime \prime}(t)+f\left(t, u(t), u^{\prime}(t)\right)=0, \quad t \in(0,+\infty), \\
u(0)=\alpha u(\eta), \quad \lim _{t \rightarrow+\infty} u^{\prime}(t)=0,
\end{gathered}
$$

where $\alpha \neq 1, \eta \in(0,+\infty)$. With the help of the established Green function and the LeraySchauder continuation theorem, suitable conditions imposed on $f$ are presented for the existence of solutions. Yan et al. [5] established the results of existence and multiplicity of positive solutions to the BVP on the half-line

$$
\begin{gathered}
u^{\prime \prime}(t)+\Phi(t) f\left(t, u, u^{\prime}\right)=0, \quad t \in(0,+\infty), \\
a u(0)-b u^{\prime}(0)=u_{0} \geq 0, \quad \lim _{t \rightarrow+\infty} u^{\prime}(t)=k>0,
\end{gathered}
$$

by using the lower and upper solutions technique. Zhang [6] researched the problem

$$
\begin{gathered}
x^{\prime \prime}(t)+q(t) f(t, x(t)), \quad t \in J_{+}, \\
x(0)=\sum_{i=1}^{m-2} \alpha_{i} x\left(\xi_{i}\right), \quad x^{\prime}(\infty)=y_{\infty},
\end{gathered}
$$

by using the fixed point theorem and the monotone iterative technique.

We note that these works [3-6] are all in real space. To the best of our knowledge, very few literatures are available for the computation of positive solutions for mutlipoint BVP on the half-line in Banach space. There are two papers we should present here. Liu [7] discussed 
the existence of solutions of the following second-order two-point BVP on infinite intervals in a Banach space $E$ :

$$
\begin{gathered}
x^{\prime \prime}(t)=f\left(t, x(t), x^{\prime}(t)\right), \quad t \in J, \\
x(0)=x_{0}, \quad x^{\prime}(\infty)=y_{\infty},
\end{gathered}
$$

where $f \in C[J \times E \times E], J=[0,+\infty), x^{\prime}(\infty)=\lim _{t \rightarrow \infty} x^{\prime}(t)$. The main tool is the Sadovskii's fixed point theorem. Zhang [8] concerned the existence of solutions of the following singular problems

$$
\begin{aligned}
& x^{\prime \prime}(t)+f\left(t, x(t), x^{\prime}(t)\right)=\theta, \quad t \in J_{+}, \\
& x(0)=\sum_{i=1}^{m-2} \alpha_{i} x\left(\xi_{i}\right), \quad x^{\prime}(\infty)=y_{\infty},
\end{aligned}
$$

where $J_{+}=(0,+\infty)$. The nonlinear term $f$ may be singular at $t=0, x=\theta$ and /or $x^{\prime}=\theta$. They used the Mönch fixed point theorem.

Motivated by the above papers, we also use the Mönch fixed point theorem to give the existence of a positive solutions of the more general BVP (1.1) for integrodifferential equations on infinite intervals in a Banach spaces. The main features of the present paper are as follows. Firstly, comparing with [3-6], the space in this paper is Banach space. The equation we discussed here is more general than those of [3-8] because the function $f$ of (1.1) has new terms $T u, S u$ and the boundary value conditions are more complicated. Moreover, the singularity of nonlinear term $f$ in this paper is more complex than $[2,7,9-11]$. Furthermore, an iterative sequence for the solution under some normal type conditions is established which makes it very important and convenient in applications. [3-7, 9-11] did not obtain this kind of result.

The rest of this paper is organized as follows. In Section 2, we give several important Lemmas. The main theorems are formulated and proved in Section 3, followed by an example in Section 4 to demonstrate the application of our results.

\section{The Preliminary and Several Lemmas}

Let

$$
\begin{gathered}
F C[J, E]=\left\{u \in C[J, E]: \sup _{t \in J} \frac{\|u(t)\|}{e^{t}}<\infty\right\}, \\
D C^{1}[J, E]=\left\{u \in C^{1}[J, E]: \sup _{t \in J} \frac{\|u(t)\|}{e^{t}}<\infty, \sup _{t \in J} \frac{\left\|u^{\prime}(t)\right\|}{e^{t}}<\infty\right\} .
\end{gathered}
$$

Evidently, $C^{1}[J, E] \subset C[J, E], D C^{1}[J, E] \subset F C[J, E]$. It is easy to see that $F C[J, E]$ is a Banach space with norm $\|u\|_{F}=\sup _{t \in J}\|u(t)\| / e^{t}$ and $D C^{1}[J, E]$ is also a Banach space with norm $\|u\|_{D}=\max \left\{\|u\|_{F},\left\|u^{\prime}\right\|_{C}\right\}$, where $\left\|u^{\prime}\right\|_{C}=\sup _{t \in J}\left\|u^{\prime}(t)\right\| / e^{t}$. 
Let

$$
\begin{gathered}
P(F)=\{u \in F C[J, E]: u(t) \geq \theta, \forall t \in J\}, \\
P(D)=\left\{u \in D C^{1}[J, E]: u(t) \geq \theta, u^{\prime}(t) \geq \theta, \forall t \in J\right\} .
\end{gathered}
$$

It is clear that $P(F), P(D)$ are cones in $F C[J, E]$ and $D C^{1}[J, E]$, respectively. A map $u \in$ $D C^{1}[J, E] \cap C^{2}\left[J_{+}, E\right]$ is called a positive solutions of the BVP (1.1) if $u \in P(D)$ and satisfies (1.1).

Let $\alpha, \alpha_{D}$ denote the Kuratowski measure of noncompactness in $E$ and $D C^{1}[J, E]$, respectively. For details on the definition and properties of the measure of noncompactness, the reader is referred to $[12,13]$.

Some conditions to be used throughout the rest of the paper are listed below.

$$
\begin{gathered}
\lim _{t \rightarrow \infty}\left(e^{-t} \int_{0}^{t} K(t, s) e^{s} d s\right)=0, \quad \int_{0}^{\infty} H(t, s) e^{s} d s<\infty, \quad \forall t \in J, \\
\lim _{t \rightarrow \infty}\left(e^{-t} \int_{0}^{\infty} H(t, s) e^{s} d s\right)=0, \quad \lim _{t^{\prime} \rightarrow t} \int_{0}^{\infty}\left|H\left(t^{\prime}, s\right)-H(t, s)\right| e^{s} d s=0, \quad \forall t, t^{\prime} \in J .
\end{gathered}
$$

In this case, let

$$
k^{*}=\sup _{t \in J}\left(e^{-t} \int_{0}^{t} K(t, s) e^{s} d s\right), \quad h^{*}=\sup _{t \in J}\left(e^{-t} \int_{0}^{\infty} H(t, s) e^{s} d s\right) .
$$

$\left(H_{2}\right) f \in C\left[J_{+} \times P_{0 \curlywedge} \times P_{0 \curlywedge} \times P \times P, P\right]$, for any $\mathcal{\lambda}>0$ and there exist $m, p, q \in L\left[J_{+}, J\right]$ and $g \in C\left[J_{+} \times J_{+} \times J \times J, J\right]$ such that

$$
\begin{gathered}
\left\|f\left(t, u_{0}, u_{1}, u_{2}, u_{3}\right)\right\| \leq m(t)+p(t) g\left(\left\|u_{0}\right\|,\left\|u_{1}\right\|,\left\|u_{2}\right\|,\left\|u_{3}\right\|\right), \quad \forall t \in J_{+}, u_{0}, u_{1} \in P_{0}, u_{2}, u_{3} \in P, \\
\frac{\left\|f\left(t, u_{0}, u_{1}, u_{2}, u_{3}\right)\right\|}{q(t)\left(\left\|u_{0}\right\|+\left\|u_{1}\right\|+\left\|u_{2}\right\|+\left\|u_{3}\right\|\right)} \longrightarrow 0 \\
\left(u_{0}, u_{1} \in P_{0}, u_{2}, u_{3} \in P,\left\|u_{0}\right\|+\left\|u_{1}\right\|+\left\|u_{2}\right\|+\left\|u_{3}\right\| \longrightarrow \infty\right)
\end{gathered}
$$

uniformly for $t \in J_{+}$, and

$$
m^{*}=\int_{0}^{\infty} m(t) d t<\infty, \quad p^{*}=\int_{0}^{\infty} p(t) d t<\infty, \quad q^{*}=\int_{0}^{\infty} q(t) e^{t} d t<\infty .
$$


$\left(H_{3}\right)$ For any $t \in J_{+}, R>0$ and countable sets $V_{i} \subset D C^{1}\left[J, P_{0 R}^{*}\right](i=0,1), V_{i} \subset$ $D C^{1}\left[J, P_{R}^{*}\right](i=2,3)$, there exist $L_{i} \in L[J, J](i=0,1,2,3)$ such that

$$
\begin{gathered}
\alpha\left(f\left(t, V_{0}(t), V_{1}(t), V_{2}(t), V_{3}(t)\right)\right) \leq \sum_{i=0}^{3} L_{i}(t) \alpha\left(V_{i}(t)\right), \\
L^{*}=\int_{0}^{\infty}\left[L_{0}(s)+L_{1}(s)+L_{2}(s) k^{*}+L_{3}(s) h^{*}\right] e^{s} d s<\frac{1}{4},
\end{gathered}
$$

where $P_{0 R}^{*}=\left\{u \in P: u \geq x_{0}^{*},\|u\| \leq R\right\}(i=0,1)$ and $P_{R}^{*}=\{u \in P:\|u\| \leq R\}$.

$\left(H_{4}\right) t \in J_{+}, x_{0}^{*} \leq u_{i} \leq \bar{u}_{i}(i=0,1), \theta \leq u_{2} \leq \bar{u}_{2}, \theta \leq u_{3} \leq \bar{u}_{3}$ imply

$$
f\left(t, u_{0}, u_{1}, u_{2}, u_{3}\right) \leq f\left(t, \bar{u}_{0}, \bar{u}_{1}, \bar{u}_{2}, \bar{u}_{3}\right)
$$

Lemma 2.1 (Lemma 1 see, [9]). If condition $\left(H_{1}\right)$ is satisfied, then the operators $T$ and $S$ defined by (1.2) are bounded linear operators from $F C[J, E]$ into $F C[J, E]$ and

$$
\|T\| \leq k^{*}, \quad\|S\| \leq h^{*}, \quad T(P(F)) \subset P(F), \quad S(P(F)) \subset P(F) .
$$

In what follows, we write

$$
Q=\left\{u \in D C^{1}[J, P]: u^{(i)}(t) \geq x_{0}^{*}, \forall t \in J, i=0,1\right\}
$$

Evidently, $Q$ is a closed convex set in $D C^{1}[J, E]$. We will reduce the BVP (1.1) to an integral equation in $E$. To this end, we first consider the operator $A$ defined by

$$
\begin{aligned}
(A u)(t)= & \frac{1}{\Delta}\left[\delta y_{\infty}+b \int_{0}^{\infty} f\left(s, u(s), u^{\prime}(s),(T u)(s),(S u)(s)\right) d s\right. \\
& \left.\quad+\sum_{i=1}^{n} k_{i} \int_{0}^{\xi_{i}} \int_{s}^{\infty} f\left(\tau, u(\tau), u^{\prime}(\tau),(T u)(\tau),(S u)(\tau)\right) d \tau d s\right] \\
& +t y_{\infty}+\int_{0}^{t} \int_{s}^{\infty} f\left(\tau, u(\tau), u^{\prime}(\tau),(T u)(\tau),(S u)(\tau)\right) d \tau d s, \quad u \in Q,
\end{aligned}
$$

where

$$
\Delta=a-\sum_{i=1}^{n} k_{i}>0, \quad \delta=b+\sum_{i=1}^{n} k_{i} \xi_{i}, \quad \Delta \leq \delta
$$

Lemma 2.2. If conditions $\left(H_{1}\right)$ and $\left(H_{2}\right)$ hold, then the operator A defined by (2.11) is a continuous operator from $Q$ into $Q$. 
Proof. Firstly, we will show $A(Q) \subset Q$. Let

$$
\begin{gathered}
\varepsilon_{0}=\frac{1}{2(1+\delta / \Delta) q^{*}\left(2+k^{*}+h^{*}\right)}, \\
r=\frac{\left\|x_{0}^{*}\right\|}{N}>0 .
\end{gathered}
$$

By virtue of condition $\left(H_{2}\right)$, there exists a $R>r$ such that

$$
\begin{gathered}
\|f(t, x, y, z, w)\| \leq \varepsilon_{0} q(t)(\|x\|+\|y\|+\|z\|+\|w\|), \\
\forall t \in J_{+}, \quad x, y \in P_{0}, \quad z, w \in P,\|x\|+\|y\|+\|z\|+\|w\|>R, \\
\|f(t, x, y, z, w)\| \leq m(t)+M p(t), \\
\forall t \in J_{+}, \quad x, y \in P_{0}, \quad z, w \in P, \quad\|x\|+\|y\|+\|z\|+\|w\| \leq R,
\end{gathered}
$$

where

$$
M=\max \left\{g\left(u_{0}, u_{1}, u_{2}, u_{3}\right): r \leq u_{i} \leq R \quad(i=0,1), 0 \leq u_{i} \leq R \quad(i=2,3)\right\} .
$$

Hence,

$$
\begin{array}{r}
\|f(t, x, y, z, w)\| \leq \varepsilon_{0} q(t)(\|x\|+\|y\|+\|z\|+\|w\|)+m(t)+M p(t) \\
\forall t \in J_{+}, \quad x, y \in P_{0}, \quad z, w \in P .
\end{array}
$$

Let $u \in Q$. We have by (2.16) and Lemma 2.1

$$
\begin{aligned}
\| f(t, & \left.u(t), u^{\prime}(t),(T u)(t),(S u)(t)\right) \| \\
& \leq \varepsilon_{0} q(t)\left(\|u(t)\|+\left\|u^{\prime}(t)\right\|+\|(T u)(t)\|+\|(S u)(t)\|\right)+m(t)+M p(t) \\
& \leq \varepsilon_{0} q(t) e^{t}\left(\|u\|_{F}+\left\|u^{\prime}\right\|_{C}+k^{*}\|u\|_{F}+h^{*}\|u\|_{F}\right)+m(t)+M p(t) \\
& \leq \varepsilon_{0} q(t) e^{t}\left(2+k^{*}+h^{*}\right)\|u\|_{D}+m(t)+M p(t), \quad \forall t \in J_{+},
\end{aligned}
$$

which together with condition $\left(H_{2}\right)$ implies the convergence of the infinite integral

$$
\int_{0}^{\infty}\left\|f\left(t, u(t), u^{\prime}(t),(T u)(t),(S u)(t)\right)\right\| d t
$$


Thus, we have

$$
\begin{gathered}
\left\|\int_{0}^{t} \int_{s}^{\infty} f\left(\tau, u(\tau), u^{\prime}(\tau),(T u)(\tau),(S u)(\tau)\right) d \tau d s\right\| \\
\quad \leq \int_{0}^{t} \int_{s}^{\infty}\left\|f\left(\tau, u(\tau), u^{\prime}(\tau),(T u)(\tau),(S u)(\tau)\right)\right\| d \tau d s \\
\quad \leq \int_{0}^{\infty} \int_{0}^{t}\left\|f\left(\tau, u(\tau), u^{\prime}(\tau),(T u)(\tau),(S u)(\tau)\right)\right\| d s d \tau \\
\leq t \int_{0}^{\infty}\left\|f\left(\tau, u(\tau), u^{\prime}(\tau),(T u)(\tau),(S u)(\tau)\right)\right\| d \tau, \quad \forall t \in J_{+},
\end{gathered}
$$

(2.11), (2.19), and $\left(H_{2}\right)$ tell us that

$$
\begin{aligned}
\|(A u)(t)\| \leq & \frac{1}{\Delta}\left[\delta\left\|y_{\infty}\right\|+b \int_{0}^{\infty}\left\|f\left(s, u(s), u^{\prime}(s),(T u)(s),(S u)(s)\right)\right\| d s\right. \\
& \left.+\sum_{i=1}^{n} k_{i} \int_{0}^{\xi_{i}} \int_{s}^{\infty}\left\|f\left(\tau, u(\tau), u^{\prime}(\tau),(T u)(\tau),(S u)(\tau)\right)\right\| d \tau d s\right] \\
& +t\left\|y_{\infty}\right\|+\int_{0}^{t} \int_{s}^{\infty}\left\|f\left(\tau, u(\tau), u^{\prime}(\tau),(T u)(\tau),(S u)(\tau)\right)\right\| d \tau d s \\
\leq & \frac{\delta}{\Delta}\left\|y_{\infty}\right\|+t\left\|y_{\infty}\right\|+\frac{b}{\Delta} \int_{0}^{\infty}\left\|f\left(s, u(s), u^{\prime}(s),(T u)(s),(S u)(s)\right)\right\| d s \\
& +\frac{\sum_{i=1}^{n} k_{i} \xi_{i}}{\Delta} \int_{0}^{\infty}\left\|f\left(\tau, u(\tau), u^{\prime}(\tau),(T u)(\tau),(S u)(\tau)\right)\right\| d \tau \\
& +t \int_{0}^{\infty}\left\|f\left(\tau, u(\tau), u^{\prime}(\tau),(T u)(\tau),(S u)(\tau)\right)\right\| d \tau .
\end{aligned}
$$

Therefore,

$$
\begin{aligned}
\frac{\|(A u)(t)\|}{e^{t}} \leq & \frac{\delta}{\Delta}\left\|y_{\infty}\right\|+\left\|y_{\infty}\right\|+\frac{b}{\Delta} \int_{0}^{\infty}\left\|f\left(s, u(s), u^{\prime}(s),(T u)(s),(S u)(s)\right)\right\| d s \\
& +\frac{\sum_{i=1}^{n} k_{i} \xi_{i}}{\Delta} \int_{0}^{\infty}\left\|f\left(\tau, u(\tau), u^{\prime}(\tau),(T u)(\tau),(S u)(\tau)\right)\right\| d \tau \\
& +\int_{0}^{\infty}\left\|f\left(\tau, u(\tau), u^{\prime}(\tau),(T u)(\tau),(S u)(\tau)\right)\right\| d \tau
\end{aligned}
$$




$$
\begin{aligned}
& \leq\left(1+\frac{\delta}{\Delta}\right) \int_{0}^{\infty}\left\|f\left(s, u(s), u^{\prime}(s),(T u)(s),(S u)(s)\right)\right\| d s+\left(\frac{\delta}{\Delta}+1\right)\left\|y_{\infty}\right\| \\
& \leq\left(1+\frac{\delta}{\Delta}\right)\left[\varepsilon_{0} q^{*}\left(2+k^{*}+h^{*}\right)\|u\|_{D}+m^{*}+M p^{*}\right]+\left(\frac{\delta}{\Delta}+1\right)\left\|y_{\infty}\right\| \\
& \leq \frac{1}{2}\|u\|_{D}+\left(1+\frac{\delta}{\Delta}\right)\left(m^{*}+M p^{*}\right)+\left(\frac{\delta}{\Delta}+1\right)\left\|y_{\infty}\right\| .
\end{aligned}
$$

Differentiating (2.11), we get

$$
(A u)^{\prime}(t)=\int_{t}^{\infty} f\left(s, u(s), u^{\prime}(s),(T u)(s),(S u)(s)\right) d s+y_{\infty} .
$$

Hence,

$$
\begin{aligned}
\frac{\left\|(A u)^{\prime}(t)\right\|}{e^{t}} & \leq \int_{0}^{\infty}\left\|f\left(s, u(s), u^{\prime}(s),(T u)(s),(S u)(s)\right) d s\right\|+\left\|y_{\infty}\right\| \\
& \leq \varepsilon_{0} q^{*}\left(2+k^{*}+h^{*}\right)\|u\|_{D}+m^{*}+M p^{*}+\left\|y_{\infty}\right\| \\
& \leq \frac{1}{2}\|u\|_{D}+m^{*}+M p^{*}+\left\|y_{\infty}\right\|, \quad \forall t \in J .
\end{aligned}
$$

It follows from (2.21) and (2.23) that

$$
\|(A u)(t)\|_{D} \leq \frac{1}{2}\|u\|_{D}+\left(1+\frac{\delta}{\Delta}\right)\left(m^{*}+M p^{*}\right)+\left(\frac{\delta}{\Delta}+1\right)\left\|y_{\infty}\right\| .
$$

So, $A u \in D C^{1}[J, E]$. On the other hand, it can be easily seen that

$$
(A u)(t) \geq \frac{\delta}{\Delta} y_{\infty} \geq y_{\infty} \geq x_{0}^{*}, \quad(A u)^{\prime}(t) \geq y_{\infty} \geq x_{0}^{*}, \quad \forall t \in J .
$$

So, $A u \in Q$. Thus, $A$ maps $Q$ into $Q$ and (2.24) holds.

Secondly, we will show that $A$ is continuous. Let $u_{m}, \bar{u} \in Q,\left\|u_{m}-\bar{u}\right\|_{D} \rightarrow 0(m \rightarrow \infty)$. Then $r=\sup _{m}\left\|u_{m}\right\|_{D}<\infty$ and $\|\bar{u}\|_{D} \leq r$. Similar to (2.21) and (2.23), it is easy to say

$$
\begin{aligned}
\left\|A u_{m}-A \bar{u}\right\|_{D} \leq\left(1+\frac{\delta}{\Delta}\right) \int_{0}^{\infty} & \| f\left(s, u_{m}(s), u_{m}^{\prime}(s),\left(T u_{m}\right)(s),\left(S u_{m}\right)(s)\right) \\
& -f\left(s, \bar{u}(s), \bar{u}^{\prime}(s),(T \bar{u})(s),(S \bar{u})(s)\right) \| d s .
\end{aligned}
$$

So we get

$$
\begin{array}{r}
f\left(s, u_{m}(s), u_{m}^{\prime}(s),\left(T u_{m}\right)(s),\left(S u_{m}\right)(s)\right) \longrightarrow f\left(s, \bar{u}(s), \bar{u}^{\prime}(s),(T \bar{u})(s),(S \bar{u})(s)\right) \\
\text { as } m \longrightarrow \infty, \quad \forall t \in J_{+} .
\end{array}
$$


Then we know from (2.17) that

$$
\begin{aligned}
& \left\|f\left(s, u_{m}(s), u_{m}^{\prime}(s),\left(T u_{m}\right)(s),\left(S u_{m}\right)(s)\right)-f\left(s, \bar{u}(s), \bar{u}^{\prime}(s),(T \bar{u})(s),(S \bar{u})(s)\right)\right\| \\
& \quad \leq 2\left[\varepsilon_{0} q(t) e^{t}\left(2+k^{*}+h^{*}\right) r+m(t)+M p(t)\right] \\
& \quad:=\sigma(t) \in L\left[J_{+}, J\right], \quad m=1,2,3, \ldots, \forall t \in J_{+} .
\end{aligned}
$$

It follows from (2.27) and (2.28) and the dominated convergence theorem that

$$
\begin{aligned}
\lim _{m \rightarrow \infty} \int_{0}^{\infty} \| f\left(s, u_{m}(s), u_{m}^{\prime}(s),\left(T u_{m}\right)(s),\left(S u_{m}\right)(s)\right) \\
-f\left(s, \bar{u}(s), \bar{u}^{\prime}(s),(T \bar{u})(s),(S \bar{u})(s)\right) \|=0
\end{aligned}
$$

It follows from (2.26) and (2.29) that $\left\|A u_{m}-A \bar{u}\right\|_{D} \rightarrow 0$ as $m \rightarrow \infty$, and the continuity of $A$ is proved.

Lemma 2.3. Let conditions $\left(H_{1}\right)$ and $\left(H_{2}\right)$ be satisfied. Then $u \in Q \cap C^{2}\left[J_{+}, E\right]$ is a solution of the $B V P$ (1.1) if and only if $u \in Q$ is a solution of the following integral equation:

$$
\begin{aligned}
u(t)=\frac{1}{\Delta}\left[\delta y_{\infty}+b \int_{0}^{\infty} f\left(s, u(s), u^{\prime}(s),(T u)(s),(S u)(s)\right) d s\right. & \\
& \left.+\sum_{i=1}^{n} k_{i} \int_{0}^{\xi_{i}} \int_{s}^{\infty} f\left(\tau, u(\tau), u^{\prime}(\tau),(T u)(\tau),(S u)(\tau)\right) d \tau d s\right] \\
& +t y_{\infty}+\int_{0}^{t} \int_{s}^{\infty} f\left(\tau, u(\tau), u^{\prime}(\tau),(T u)(\tau),(S u)(\tau)\right) d \tau d s .
\end{aligned}
$$

Proof. Integrating the differential equation in (1.1) from $t$ to $\infty$, one has

$$
u^{\prime}(t)=y_{\infty}+\int_{t}^{\infty} f\left(s, u(s), u^{\prime}(s),(T u)(s),(S u)(s)\right) d s
$$

Then, integrating (2.31) from 0 to $t$, we have

$$
u(t)=u(0)+t y_{\infty}+\int_{0}^{t} \int_{s}^{\infty} f\left(\tau, u(\tau), u^{\prime}(\tau),(T u)(\tau),(S u)(\tau)\right) d \tau d s
$$

By Lemma 2.2, we know that $\int_{0}^{t} \int_{S}^{\infty} f\left(\tau, u(\tau), u^{\prime}(\tau),(T u)(\tau),(S u)(\tau)\right) d \tau d s$ is convergent. Since $a u(0)-b u^{\prime}(0)-\sum_{i=1}^{n} k_{i} u\left(\xi_{i}\right)=\theta$, we can compute those coefficients $u(0), u^{\prime}(0), u\left(\xi_{i}\right)$ and then obtain (2.30).

Conversely, if $u$ is a solution of integral equation (2.30), then direct differentiation gives the proof. 
Lemma 2.4 (Mönch Fixed Point Theorem [10]). Let $Q$ be a closed convex set of $E$ and $u \in Q$. Assume that the continuous operator $A: Q \rightarrow Q$ has the following property: $V \subset$ $\overline{c o}(\{u\} \cup F(V)) \Rightarrow V$ is relatively compact. Then $F$ has a fixed point in $Q$.

Lemma 2.5. Let $H$ be a bounded set in $D C^{1}[J, E]$. Suppose that $H^{\prime}(t)$ is equicontinuous on any finite subinterval $[0, c](c>0)$ of $J$ and $e^{-t}\left\|u^{(i)}\right\| \rightarrow 0$ as $t \rightarrow \infty$ uniformly for $u \in H(i=0,1)$. Then

$$
\alpha_{D}(H)=\max \left\{\sup _{t \in J}\left[e^{-t} \alpha(H(t))\right], \sup _{t \in J}\left[e^{-t} \alpha\left(H^{(1)}(t)\right)\right]\right\}
$$

where $H^{(i)}(t)=\left\{\left(x_{m}\right)^{(i)}(t): x_{m} \in H, m=1,2,3, \ldots\right\}(i=0,1), \alpha$ and $\alpha_{D}$ denote the Kuratowski measure of noncompactness of bounded sets in $E$ and $D C^{1}[J, E]$, respectively.

Proof. The proof is similar to [11, Lemma 7], we omit it.

Lemma 2.6. If condition $\left(H_{4}\right)$ is satisfied, then $x, y \in Q, x^{(i)} \leq y^{(i)}, t \in J(i=0,1)$ imply that $(A x)^{(i)} \leq(A y)^{(i)}, t \in J(i=0,1)$.

Proof. It is easy to see that this lemma follows from (2.11) and (2.22) and condition $\left(H_{4}\right)$.

\section{Main Results}

In the following, we will give the main results of this paper.

Theorem 3.1. Assume that $\left(H_{1}\right)-\left(H_{3}\right)$ hold, then the BVP (1.1) has a positive solution $\bar{u} \in$ $D C^{1}[J, E] \cap C^{2}\left[J_{+}, E\right]$ satisfying $(\bar{u})^{(i)}(t) \geq x_{0}^{*}$ for $t \in J(i=0,1)$.

Proof. By Lemma 2.2, the operator $A$ defined by (2.11) is a continuous operator from $Q$ into $Q$. And, by Lemma 2.3, we only need to show that $A$ has a fixed point $\bar{u}$ in $Q$.

Choose

$$
R>2\left[\left(1+\frac{\delta}{\Delta}\right)\left(m^{*}+M p^{*}\right)+\left(\frac{\delta}{\Delta}+1\right)\left\|y_{\infty}\right\|\right]
$$

and let $Q_{1}=\left\{u \in Q:\|u\|_{D} \leq R\right\}$. Obviously, $Q_{1}$ is a bounded closed convex set in the space $D C^{1}[J, E]$. It is easy to see that $Q_{1}$ is not empty since $(\delta / \Delta+1) y_{\infty} \in Q_{1}$. It follows from (2.24) and (3.1) that $u \in Q_{1}$ implies $A u \in Q_{1}$, that is, $A$ maps $Q_{1}$ into $Q_{1}$.

Now, we are in position to show that $A\left(Q_{1}\right)$ is relatively compact. Let $V=\left\{u_{m}: m=\right.$ $1,2, \ldots\} \subset Q_{1}$ satisfying $V \subset \overline{c o}(\{u\} \cup\{(A V)\})$ for some $u \in Q_{1}$. Then $\left\|u_{m}\right\|_{D} \leq R$. So we have by $(2.11)$ and $(2.22)$ that 
Abstract and Applied Analysis

$$
\begin{aligned}
&\left(A u_{m}\right)(t)=\frac{1}{\Delta}\left[\delta y_{\infty}+b \int_{0}^{\infty} f\left(s, u_{m}(s), u_{m}^{\prime}(s),\left(T u_{m}\right)(s),\left(S u_{m}\right)(s)\right) d s\right. \\
&\left.+\sum_{i=1}^{n} k_{i} \int_{0}^{\xi_{i}} \int_{s}^{\infty} f\left(\tau, u_{m}(\tau), u_{m}^{\prime}(\tau),\left(T u_{m}\right)(\tau),\left(S u_{m}\right)(\tau)\right) d \tau d s\right] \\
&+t y_{\infty}+\int_{0}^{t} \int_{s}^{\infty} f\left(\tau, u_{m}(\tau), u_{m}^{\prime}(\tau),\left(T u_{m}\right)(\tau),\left(S u_{m}\right)(\tau)\right) d \tau d s \\
&= \frac{1}{\Delta}\left[\delta y_{\infty}+b \int_{0}^{\infty} f\left(s, u_{m}(s), u_{m}^{\prime}(s),\left(T u_{m}\right)(s),\left(S u_{m}\right)(s)\right) d s\right. \\
&\left.+\sum_{i=1}^{n} k_{i} \int_{0}^{\xi_{i}} \int_{s}^{\infty} f\left(\tau, u_{m}(\tau), u_{m}^{\prime}(\tau),\left(T u_{m}\right)(\tau),\left(S u_{m}\right)(\tau)\right) d \tau d s\right] \\
&+t y_{\infty}+\int_{0}^{t} s f\left(s, u_{m}(s), u_{m}^{\prime}(s),\left(T u_{m}\right)(s),\left(S u_{m}\right)(s)\right) d s \\
&+\int_{t}^{\infty} t f\left(s, u_{m}(s), u_{m}^{\prime}(s),\left(T u_{m}\right)(s),\left(S u_{m}\right)(s)\right) d s, \\
&\left(A u_{m}\right)^{\prime}(t)=y_{\infty}+\int_{t}^{\infty} f\left(s, u_{m}(s), u_{m}^{\prime}(s),\left(T u_{m}\right)(s),\left(S u_{m}\right)(s)\right) d s .
\end{aligned}
$$

So for any $t_{1}, t_{2} \in[0, c](c>0), t_{1}<t_{2}$, we have that

$$
\begin{aligned}
\left\|\left(A u_{m}\right)^{\prime}\left(t_{2}\right)-\left(A u_{m}\right)^{\prime}\left(t_{1}\right)\right\| & \leq \int_{t_{1}}^{t_{2}}\left\|f\left(s, u_{m}(s), u_{m}^{\prime}(s),\left(T u_{m}\right)(s),\left(S u_{m}\right)(s)\right)\right\| d s \\
& \leq \varepsilon_{0}\left(2+k^{*}+h^{*}\right) R \int_{t_{1}}^{t_{2}} q(s) e^{s} d s+\int_{t_{1}}^{t_{2}}[m(s)+M p(s)] d s .
\end{aligned}
$$

(3.3) implies that $\left\{\left(A u_{m}\right)^{\prime}(t)\right\}(m=1,2,3, \ldots)$ is equicontinuous on any finite subinterval of $J$.

On the other hand, by (2.17), (2.20), (3.2) we can obtain

$$
\begin{gathered}
\left\|\left(A u_{m}\right)(t)\right\| \leq \frac{1}{2}\left\|u_{m}\right\|_{D}+\left(t+\frac{\delta}{\Delta}\right)\left(m^{*}+M p^{*}\right)+\left(\frac{\delta}{\Delta}+t\right)\left\|y_{\infty}\right\|, \quad \forall t \in J_{+}, \\
\left\|\left(A u_{m}\right)^{\prime}(t)\right\| \leq \frac{1}{2}\left\|u_{m}\right\|_{D}+m^{*}+M p^{*}+\left\|y_{\infty}\right\|, \quad \forall t \in J_{+},
\end{gathered}
$$

which implies that $e^{-t}\left\|\left(A u_{m}\right)^{(i)}(t)\right\| \rightarrow 0$ as $t \rightarrow \infty(i=0,1)$ uniformly for $m=1,2,3, \ldots$ Hence, by Lemma 2.5 we have

$$
\alpha_{D}(A V)=\max \left\{\sup _{t \in J}\left[e^{-t} \alpha((A V)(t))\right], \sup _{t \in J}\left[e^{-t} \alpha\left((A V)^{(1)}(t)\right)\right]\right\}
$$


where $A V=\left\{A u_{m}: u_{m} \in V, m=1,2,3, \ldots\right\}$, and $(A V)^{(1)}=\left\{\left(A u_{m}\right)^{(1)}(t): u_{m} \in V, m=\right.$ $1,2,3, \ldots\}$.

It follows from (2.18) that the infinite integral $\int_{0}^{\infty} \| f\left(t, u_{m}(t), u_{m}^{\prime}(t),\left(T u_{m}\right)(t)\right.$, $\left.\left(S u_{m}\right)(t)\right) \| d t$ is convergence uniformly for $m=1,2,3, \ldots$. So, for any $\varepsilon>0$, we can choose a large $T>0$ such that

$$
\int_{T}^{\infty}\left\|f\left(t, u_{m}(t), u_{m}^{\prime}(t),\left(T u_{m}\right)(t),\left(S u_{m}\right)(t)\right)\right\| d t<\varepsilon
$$

holds for any $m$. Then, by $[6$, Theoerm 1.2 .3$],(3.6)$, and $\left(H_{3}\right)$, we obtain

$$
\begin{aligned}
e^{-t} \alpha((A V)(t)) \leq & 2 \frac{t}{e^{t}} \int_{t}^{T} \alpha\left(f\left(s, V(s), V^{\prime}(s),(T V)(s),(S V)(s)\right)\right) d s+2 \varepsilon \\
& +2 \frac{s}{e^{t}} \int_{0}^{t} \alpha\left(f\left(s, V(s), V^{\prime}(s),(T V)(s),(S V)(s)\right)\right) d s \\
\leq & 4 \int_{0}^{\infty} \alpha\left(f\left(s, V(s), V^{\prime}(s),(T V)(s),(S V)(s)\right)\right) d s+2 \varepsilon \\
\leq & 4 \alpha_{D}(V) \int_{0}^{\infty}\left[L_{0}(s)+L_{1}(s)+L_{2}(s) k^{*}+L_{3}(s) h^{*}\right] e^{s} d s+2 \varepsilon, \\
e^{-t} \alpha\left((A V)^{(1)}(t)\right) \leq & 2 \int_{0}^{\infty} \alpha\left(f\left(s, V(s), V^{\prime}(s),(T V)(s),(S V)(s)\right)\right) d s+2 \varepsilon \\
\leq & 2 \alpha_{D}(V) \int_{0}^{\infty}\left[L_{0}(s)+L_{1}(s)+L_{2}(s) k^{*}+L_{3}(s) h^{*}\right] e^{s} d s+2 \varepsilon
\end{aligned}
$$

By (3.7) and noting the fact that $\varepsilon>0$ is arbitrary, we see that

$$
a_{D}(A V) \leq 4 L^{*} a_{D}(V) \leq a_{D}(V) .
$$

On the other hand, $\alpha_{D}(V) \leq \alpha_{D}\{\overline{c o}(\{u\} \bigcup(A V))\}=\alpha_{D}(A V)$. Then $\alpha_{D}(V)=0$, that is, $V$ is relatively compact in $D C^{1}[J, E]$.

Hence, the Mönch fixed point theorem implies that $A$ has a fixed point $\bar{u}$ in $Q_{1}$ and this theorem is proved.

Theorem 3.2. Let cone $P$ be normal and let conditions $\left(H_{1}\right)-\left(H_{4}\right)$ be satisfied. Then the BVP (1.1) has a positive solution $y \in Q \cap\left[J_{+}, E\right]$ which is minimal in the sense that $u^{(i)}(t) \geq y^{(i)}(t), t \in J(i=$ $0,1)$ for any positive solution $u \in Q \cap\left[J_{+}, E\right]$. Moreover, $\|y\|_{D} \leq 2 \gamma+\left\|x_{0}\right\|_{D}$, where

$$
r=\left(1+\frac{\delta}{\Delta}\right)\left(m^{*}+M p^{*}\right)+\left(\frac{\delta}{\Delta}+1\right)\left\|y_{\infty}\right\|
$$


and there exists a monotone iterative sequence $\left\{u_{m}\right\}$ such that $u_{m}^{(i)} \rightarrow y^{(i)}$ as $m \rightarrow \infty(i=0,1)$ uniformly on $J$ and $u_{m}^{\prime \prime}(t) \rightarrow y^{\prime \prime}(t)$ as $m \rightarrow \infty$ for any $t \in J_{+}$, where

$$
\begin{aligned}
u_{0}(t)= & \frac{1}{\Delta}\left[\delta y_{\infty}+b \int_{0}^{\infty} f\left(s, x_{0}^{*}, x_{0}^{*}, \theta, \theta\right) d s+\sum_{i=1}^{n} k_{i} \int_{0}^{\xi_{i}} \int_{s}^{\infty} f\left(\tau, x_{0}^{*}, x_{0}^{*}, \theta, \theta\right) d \tau d s\right] \\
& +t y_{\infty}+\int_{0}^{t} \int_{s}^{\infty} f\left(\tau, x_{0}^{*}, x_{0}^{*}, \theta, \theta\right) d \tau d s, \\
u_{m}(t)= & \frac{1}{\Delta}\left[\delta y_{\infty}+b \int_{0}^{\infty} f\left(s, u_{m-1}(s), u_{m-1}^{\prime}(s),\left(T u_{m-1}\right)(s),\left(S u_{m-1}\right)(s)\right) d s\right. \\
& \left.+\sum_{i=1}^{n} k_{i} \int_{0}^{\xi_{i}} \int_{s}^{\infty} f\left(\tau, u_{m-1}(\tau), u_{m-1}^{\prime}(\tau),\left(T u_{m-1}\right)(\tau),\left(S u_{m-1}\right)(\tau)\right) d \tau d s\right] \\
& +t y_{\infty}+\int_{0}^{t} \int_{s}^{\infty} f\left(\tau, u_{m-1}(\tau), u_{m-1}^{\prime}(\tau),\left(T u_{m-1}\right)(\tau),\left(S u_{m-1}\right)(\tau)\right) d \tau d s .
\end{aligned}
$$

Proof. From (3.10), we can find that $u_{0} \in C[J, E]$ and

$$
u_{0}^{\prime}(t)=\int_{t}^{\infty} f\left(s, x_{0}^{*}, x_{0}^{*}, \theta, \theta\right) d s+y_{\infty}
$$

By (3.10) and (3.12), we have $u_{0}^{(i)} \geq y_{\infty} \geq x_{0}^{*}(i=0,1)$ and

$$
\begin{aligned}
\left\|u_{0}(t)\right\| \leq & \frac{1}{\Delta}\left[\delta\left\|y_{\infty}\right\|+b \int_{0}^{\infty}\left\|f\left(s, x_{0}^{*}, x_{0}^{*}, \theta, \theta\right)\right\| d s+\sum_{i=1}^{n} k_{i} \int_{0}^{\xi_{i}} \int_{s}^{\infty}\left\|f\left(\tau, x_{0}^{*}, x_{0}^{*}, \theta, \theta\right)\right\| d \tau d s\right] \\
& +t\left\|y_{\infty}\right\|+\int_{0}^{t} \int_{s}^{\infty}\left\|f\left(\tau, x_{0}^{*}, x_{0}^{*}, \theta, \theta\right)\right\| d \tau d s \\
\leq & \frac{\delta}{\Delta}\left\|y_{\infty}\right\|+t\left\|y_{\infty}\right\|+\frac{b}{\Delta} \int_{0}^{\infty}\left\|f\left(s, x_{0}^{*}, x_{0}^{*}, \theta, \theta\right)\right\| d s \\
& +\frac{\sum_{i=1}^{n} k_{i} \xi_{i}}{\Delta} \int_{0}^{\infty}\left\|f\left(\tau, x_{0}^{*}, x_{0}^{*}, \theta, \theta\right)\right\| d \tau+t \int_{0}^{\infty}\left\|f\left(\tau, x_{0}^{*}, x_{0}^{*}, \theta, \theta\right)\right\| d \tau, \\
\left\|u_{0}^{\prime}(t)\right\| \leq & \int_{t}^{\infty}\left\|f\left(s, x_{0}^{*}, x_{0}^{*}, \theta, \theta\right)\right\| d s+\left\|y_{\infty}\right\| .
\end{aligned}
$$

(2.18) implies that $e^{-t}\left\|u_{0}^{(i)}\right\| \rightarrow 0$ as $t \rightarrow \infty(i=0,1)$. Hence, $\left\|u_{0}\right\|_{F},\left\|u_{0}\right\|_{C}<\infty(i=0,1)$, which implies $u_{0} \in D C^{1}[J, E]$. From (2.11) and (3.11) we get

$$
u_{m}(t)=\left(A u_{m-1}\right)(t), \quad \forall t \in J, m=1,2,3, \ldots
$$


By Lemma 2.2, we get $u_{m} \in Q$ and

$$
\left\|u_{m}(t)\right\|_{D}=\left\|\left(A u_{m-1}\right)(t)\right\|_{D} \leq \frac{1}{2}\left\|u_{m-1}\right\|_{D}+\gamma, \quad m=1,2,3, \ldots,
$$

By Lemma 2.6 and (3.15), we have

$$
u_{0}^{*} \leq u_{0}^{(i)}(t) \leq u_{1}^{(i)}(t) \leq \cdots \leq u_{m}^{(i)}(t) \leq \cdots, \quad \forall t \in J(i=0,1) .
$$

It follows from (3.15), by induction, that

$$
\left\|u_{m}(t)\right\|_{D} \leq \gamma+\frac{1}{2} \gamma+\cdots\left(\frac{1}{2}\right)^{m-1} \gamma+\left(\frac{1}{2}\right)^{m}\left\|u_{0}\right\|_{D} \leq 2 \gamma+\left\|u_{0}\right\|_{D^{\prime}} \quad m=1,2,3, \ldots
$$

Let $K=\left\{u \in Q:\|u\| \leq 2 \gamma+\left\|u_{0}\right\|_{D}\right\}$. So, $K$ is a bounded closed convex set in the space $D C^{1}[J, E]$ and operator $A$ maps $K$ into $K$. Clearly, $K$ is not empty since $u_{0} \in K$.

Let $W=\left\{u_{m}: m=0,1,2, \ldots\right\}$ and $A W=\left\{A u_{m}: m=0,1,2, \ldots\right\}$. Obviously, $W \subset K$ and $W=\left\{u_{0}\right\} \bigcup(A W)$. Similarly, as the proof in Theorem 3.1, we can obtain $\alpha_{D}(W)=0$, that is, $W$ is relatively compact in $D C^{1}[J, E]$. So there exist a $y \in D C^{1}[J, E]$ and a subsequence $\left\{u_{m_{j}}: j=1,2,3, \ldots\right\} \subset W$ such that $\left\{u_{m_{j}}^{(i)}: j=1,2,3, \ldots\right\}$ converges to $y^{(i)}(t)$ uniformly on $J(i=0,1)$. Since that $P$ is normal and $\left\{u_{m}^{(i)}(t): m=1,2,3, \ldots\right\}$ is nondecreasing on account of (3.16), it is easy to see that the entire sequence $\left\{u_{m}^{(i)}(t): m=1,2,3, \ldots\right\}$ converges to $y^{(i)}(t)$ uniformly on $J(i=0,1)$. By $u_{m} \in K$ and $K$ is a closed convex set in space $D C^{1}[J, E]$, we have $y \in K$. Let $t \in J$ be arbitrarily fixed. It is clear that

$$
H(t, s)\left\|u_{m}(s)-y(s)\right\| \longrightarrow 0, \quad \text { as } m \longrightarrow \infty, \forall s \in J
$$

and, by (3.17), we have

$$
H(t, s)\left\|u_{m}(s)-y(s)\right\| \leq 2\left(2 \gamma+\left\|u_{0}\right\|_{D}\right) H(t, s) e^{s}=\rho(s) \in L[J, J], \quad \forall s \in J(m=1,2, \ldots)
$$

It follows from (3.18) and (3.19) and the dominated convergence theorem that

$$
\left\|\left(S u_{m}\right)(s)-(S y)(s)\right\| \leq \int_{0}^{\infty} H(t, s)\left\|u_{m}(s)-y(s)\right\| d s \longrightarrow 0 \quad(m \longrightarrow \infty),
$$

which implies that $\left(S u_{m}\right)(t) \rightarrow(S y)(t)$, as $m \rightarrow \infty$ for any $t \in J$. Hence,

$$
\begin{array}{r}
f\left(s, u_{m}(s), u_{m}^{\prime}(s),\left(T u_{m}\right)(s),\left(S u_{m}\right)(s)\right) \longrightarrow f\left(s, y(s), y^{\prime}(s),(T y)(s),(S y)(s)\right) \\
\text { as } m \longrightarrow \infty, \quad \forall s \in J_{+} .
\end{array}
$$


By virtue of (3.17) and Lemma 2.1, we have

$$
\begin{aligned}
\| f(s, & \left.u_{m}(s), u_{m}^{\prime}(s),\left(T u_{m}\right)(s),\left(S u_{m}\right)(s)\right)-f\left(s, y(s), y^{\prime}(s),(T y)(s),(S y)(s)\right) \| \\
& \leq 2\left[\varepsilon_{0} q(s) e^{s}\left(2+k^{*}+h^{*}\right)\left\|u_{m}\right\|_{D}+m(s)+M p(s)\right] \\
& \leq 2\left[\varepsilon_{0} q(s) e^{s}\left(2+k^{*}+h^{*}\right)\left(2 \gamma+\left\|u_{0}\right\|_{D}\right)+m(s)+M p(s)\right] .
\end{aligned}
$$

Now, noting (3.21) and (3.22) and taking limit as $m \rightarrow \infty$ in (3.11), we obtain

$$
\begin{aligned}
y(t)=\frac{1}{\Delta}\left[\delta y_{\infty}+b \int_{0}^{\infty} f\left(s, y(s), y^{\prime}(s),(T y)(s),(S y)(s)\right) d s\right. & \\
& \left.+\sum_{i=1}^{n} k_{i} \int_{0}^{\xi_{i}} \int_{s}^{\infty} f\left(\tau, y(\tau), y^{\prime}(\tau),(T y)(\tau),(S y)(\tau)\right) d \tau d s\right] \\
& +t y_{\infty}+\int_{0}^{t} \int_{s}^{\infty} f\left(\tau, y(\tau), y^{\prime}(\tau),(T y)(\tau),(S y)(\tau)\right) d \tau d s
\end{aligned}
$$

which follows from Lemma 2.3 that $y \in K \cap C^{2}\left[J_{+}, E\right]$ and $y(t)$ is positive solution of the BVP (1.1). Differentiating (3.11) twice, we get

$$
u_{m}^{\prime \prime}=-f\left(s, u_{m}(s), u_{m}^{\prime}(s),\left(T u_{m}\right)(s),\left(S u_{m}\right)(s)\right), \quad \forall s \in J_{+}, m=1,2,3, \ldots
$$

Hence, by (3.21), we obtain

$$
\lim _{m \rightarrow \infty} u_{m}^{\prime \prime}=-f\left(s, y(s), y^{\prime}(s),(T y)(s),(S y)(s)\right), \quad \forall s \in J_{+} .
$$

Let $u(t)$ be any other positive solution of the BVP (1.1). By Lemma 2.3, we have $u(t) \in$ $Q$ and $u(t)=(A u)(t)$, for $t \in J$. It is clear that $u^{(i)}(t) \geq x_{0}^{*}>0$ for any $t \in J(i=0,1)$. So, by Lemma 2.6, we can have $u^{(i)}(t) \geq u_{0}^{(i)}$ for any $t \in J(i=0,1)$. Assume that $u^{(i)} \geq u_{m-1}^{(i)}(t)$ for any $t \in J, m \geq 1(i=0,1)$. Then, it follows from Lemma 2.6 that $(A u)^{(i)} \geq\left(A u_{m-1}\right)^{(i)}(t)$ for any $t \in J,(i=0,1)$, that is, $u^{(i)}(t) \geq u_{m}^{(i)}(t)$ for any $t \in J,(i=0,1)$. Hence, by induction, we get

$$
u^{(i)}(t) \geq u_{m}^{(i)}(t), \quad \forall t \in J,(i=0,1 ; m=0,1,2,3, \ldots) .
$$

Now, taking limits as $m \rightarrow \infty$ in (3.26), we get $u^{(i)}(t) \geq y^{(i)}(t)$ for $t \in J(i=0,1)$, and this completes the proof.

Theorem 3.3. Let cone $P$ be fully regular and let conditions $\left(H_{1}\right),\left(H_{2}\right),\left(H_{4}\right)$ be satisfied. Then the conclusion of Theorem 3.2 holds also.

Proof. The proof is almost the same as that of Theorem 3.2. The only difference is that, instead of using condition $\left(H_{3}\right)$, the conclusion $\alpha_{D}(W)=0$ was implied directly by (3.16) and (3.17) and full regularity of $P$ and Lemma 2.5. 


\section{An Example}

Example 4.1. Consider the following infinite system of scalar second-order multipoint singular integrodifferential equation:

$$
\begin{aligned}
&-u_{n}^{\prime \prime}(t)= \frac{e^{-2 t}}{n^{2} \sqrt{t}}\left(1+u_{n}+u_{2 n}^{\prime}+\frac{1}{2 n^{2} u_{n}(t)}+\frac{1}{8 n^{3} u_{2 n}^{\prime}(t)}\right)^{1 / 2} \\
&+\frac{e^{-3 t}}{\sqrt{n t}}\left(\int_{0}^{t} \frac{e^{-s(t+1)} u_{n}(s) d s}{(1+t s+s)^{3}}\right)^{1 / 3} \\
&+\frac{e^{-4 t}}{n \sqrt{t}(1+t)}\left(\int_{0}^{\infty} e^{-2 s} \cos ^{2}(t-s) u_{2 n}(s) d s\right)^{1 / 5}, \quad \forall t \in J_{+}, \\
& 80 u_{n}(0)-3 u_{n}^{\prime}(0)-\sum_{i=1}^{7} i u_{n}\left(\frac{i+3}{4}\right)=0, \quad \lim _{t \rightarrow+\infty} u_{n}^{\prime}(t)=\frac{1}{n^{2}} .
\end{aligned}
$$

Conclusion. Infinite system (4.2) has a minimal positive solution $u_{n}(t)$ satisfying $u_{n}(t), u_{n}^{\prime}(t) \geq$ $1 / n^{2}$ for $0 \leq t \leq+\infty(n=1,2,3, \ldots)$, and this minimal solution can be obtained by taking limits from some iterative sequences.

Proof. Let $E=l^{1}=\left\{u=\left(u_{1}, u_{2}, \ldots, u_{n}, \ldots\right): \sum_{n=1}^{\infty}\left|u_{n}\right|<\infty\right\}$ with the norm $\|u\|=\sum_{n=1}^{\infty}\left|u_{n}\right|$. Choose $P=\left\{u=\left(u_{n}\right) \in l^{1}: u_{n} \geq 0, n=1,2, \ldots\right\}$. It is easy to see that $E$ is weakly sequence complete, and $P$ is a normal cone in $E$. Thus, $P$ is fully regular.

Now we consider the infinite system (4.2), which can be regarded as the BVP (1.1) with $a=80, b=3, k_{i}=i, \xi_{i}=(i+3) / 4(i=1,2, \ldots, 7), y_{\infty}=(1,1 / 4,1 / 9, \ldots)$. So we have

$$
\Delta=80-\sum_{i=1}^{7} i=52, \quad \delta=3+\sum_{i=1}^{7} i \frac{i+3}{4}=59, \quad \Delta \leq \delta .
$$

In this situation,

$$
\begin{gathered}
u=\left(u_{1}, u_{2}, \ldots, u_{n}, \ldots\right), \quad v=\left(v_{1}, v_{2}, \ldots, v_{n}, \ldots\right), \\
w=\left(w_{1}, w_{2}, \ldots, w_{n}, \ldots\right), \quad z=\left(z_{1}, z_{2}, \ldots, z_{n}, \ldots\right), \\
K(t, s)=\frac{e^{-s(t+1)}}{(1+t s+s)^{3}}, \quad H(t, s)=e^{-2 s} \cos ^{2}(t-s), \\
f=\left(f_{1}, f_{2}, \ldots, f_{n}, \ldots\right),
\end{gathered}
$$


in which

$$
\begin{aligned}
f_{n}(t, u, v, w, z)= & \frac{e^{-2 t}}{n^{2} \sqrt{t}}\left(1+u_{n}+v_{2 n}+\frac{1}{2 n^{2} u_{n}(t)}+\frac{1}{8 n^{3} v_{2 n}(t)}\right)^{1 / 2} \\
& +\frac{e^{-3 t}}{\sqrt{n t}} w_{n}^{1 / 3}+\frac{e^{-4 t}}{n \sqrt{t}(1+t)} z_{2 n}^{1 / 5} .
\end{aligned}
$$

Let $x_{0}^{*}=y_{\infty}=(1,1 / 4,1 / 9, \ldots)$. Then $P_{0 \curlywedge}=\left\{u=\left(u_{1}, u_{2}, \ldots, u_{n}, \ldots\right): u_{n} \geq \lambda / n^{2}, n=1,2, \ldots\right\}$ for any $\lambda>0$ and the condition $\left(H_{1}\right)$ holds for $k^{*}<1 / 2$ and $h^{*}<1$. It is clear that $f \in$ $C\left[J_{+} \times P_{0 \lambda} \times P_{0 \lambda} \times P \times P, P\right]$ for any $\lambda>0$. By (4.4) we get

$$
\|f(t, u, v, w, z)\| \leq \frac{e^{-2 t}}{\sqrt{t}}\left\{\left(\frac{11}{4}+\|u\|+\|v\|\right)^{1 / 2}+\|w\|^{1 / 3}+\|z\|^{1 / 5}\right\} .
$$

So, the condition $\left(H_{2}\right)$ is satisfied for $m(t)=0, p(t)=q(t)=e^{-2 t} / \sqrt{t}$ and

$$
g\left(u_{0}, u_{1}, u_{2}, u_{3}\right)=\left(\frac{11}{4}+u_{0}+u_{1}\right)^{1 / 2}+u_{2}^{1 / 3}+u_{3}^{1 / 5}
$$

It is easy to see that $\left(H_{4}\right)$ holds. Thus, our conclusion follows from Theorem 3.3 immediately.

Remark 4.2. It is easy to see that the Example 4.1 cannot be solved by [3-11, 13].

\section{Acknowledgments}

The authors are grateful to the anonymous referees for their helpful suggestions and comments. Z. Hao acknowledges support from NSFC (10771117). Ph.D. Programs Foundation of Ministry of Education of China (20093705120002) and NSF of Shandong Province of China (Y2008A24). China Postdoctoral Science Foundation (20090451290), ShanDong Province Postdoctoral Foundation (200801001), and Foundation of Qufu Normal University (BSQD07026).

\section{References}

[1] S. Z. Chen and Y. Zhang, "Singular boundary value problems on a half-line," Journal of Mathematical Analysis and Applications, vol. 195, no. 2, pp. 449-468, 1995.

[2] D. O'Regan, Theory of Singular Boundary Value Problems, World Scientific Publishing, River Edge, NJ, USA, 1994.

[3] Y. Liu, "Existence and unboundedness of positive solutions for singular boundary value problems on half-line," Applied Mathematics and Computation, vol. 144, no. 2-3, pp. 543-556, 2003.

[4] H. Lian and W. Ge, "Solvability for second-order three-point boundary value problems on a half-line," Applied Mathematics Letters, vol. 19, no. 10, pp. 1000-1006, 2006.

[5] B. Yan, D. O'Regan, and R. P. Agarwal, "Unbounded solutions for singular boundary value problems on the semi-infinite interval: upper and lower solutions and multiplicity," Journal of Computational and Applied Mathematics, vol. 197, no. 2, pp. 365-386, 2006. 
[6] X. Zhang, "Successive iteration and positive solutions for a second-order multi-point boundary value problem on a half-line," Computers E Mathematics with Applications, vol. 58, no. 3, pp. 528-535, 2009.

[7] Y. Liu, "Boundary value problems for second order differential equations on unbounded domains in a Banach space," Applied Mathematics and Computation, vol. 135, no. 2-3, pp. 569-583, 2003.

[8] X. Zhang, "Existence of positive solutions for multi-point boundary value problems on infinite intervals in Banach spaces," Applied Mathematics and Computation, vol. 206, no. 2, pp. 932-941, 2008.

[9] D. Guo, "Multiple positive solutions for $n$ th-order impulsive integro-differential equations in Banach spaces," Nonlinear Analysis: Theory, Methods E Applications, vol. 60, no. 5, pp. 955-976, 2005.

[10] D. Guo, V. Lakshmikantham, and X. Liu, Nonlinear Integral Equations in Abstract Spaces, vol. 373 of Mathematics and Its Applications, Kluwer Academic Publishers, Dordrecht, The Netherlands, 1996.

[11] D. Guo, "Existence of solutions for nth order impulsive integro-differential equations in a banach space," Nonlinear Analysis, Theory, Methods and Applications, vol. 47, no. 2, pp. 741-752, 2001.

[12] K. Deimling, Ordinary Differential Equations in Banach Spaces, vol. 596 of Lecture Notes in Mathematics, Springer, Berlin, Germany, 1977.

[13] V. Lakshmikantham and S. Leela, Nonlinear Differential Equations in Abstract Spaces, vol. 2 of International Series in Nonlinear Mathematics: Theory, Methods and Applications, Pergamon Press, Oxford, UK, 1981. 


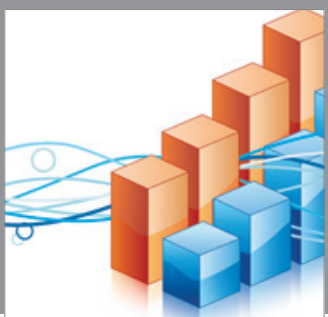

Advances in

Operations Research

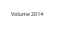

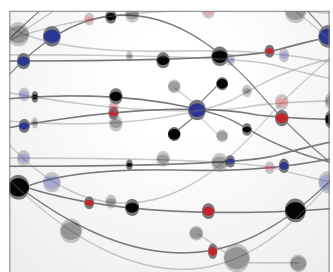

\section{The Scientific} World Journal
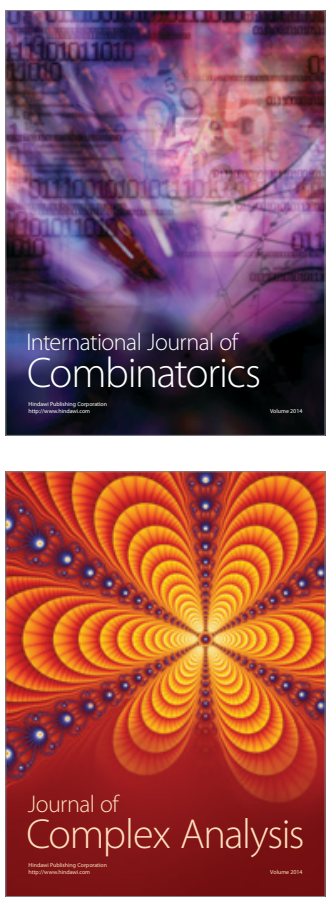

International Journal of

Mathematics and

Mathematical

Sciences
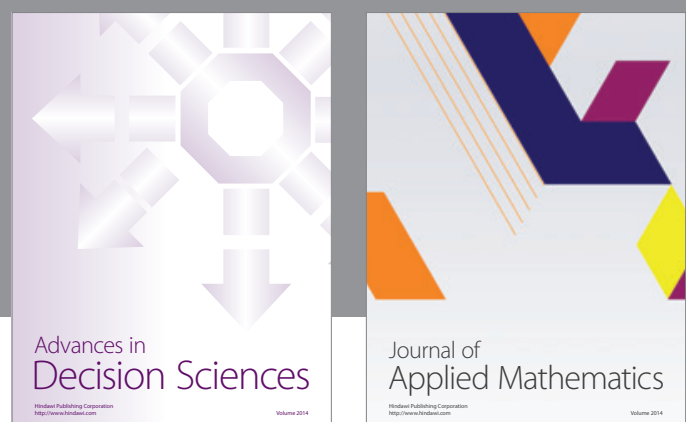

Journal of

Applied Mathematics
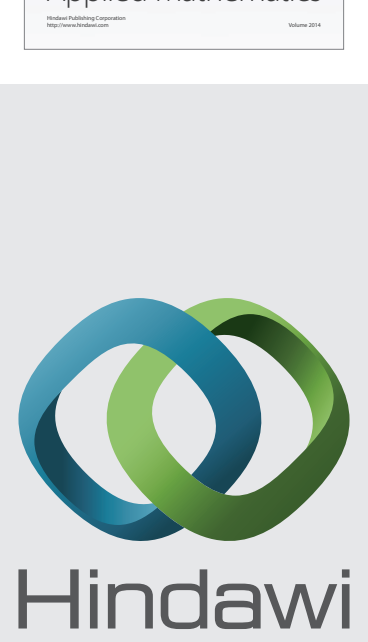

Submit your manuscripts at http://www.hindawi.com
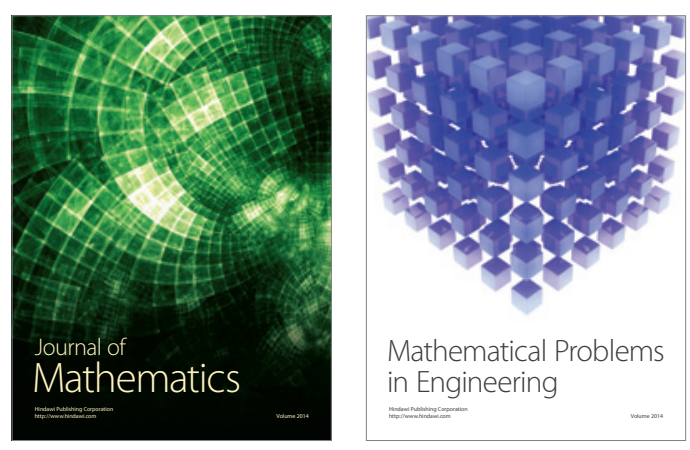

Mathematical Problems in Engineering
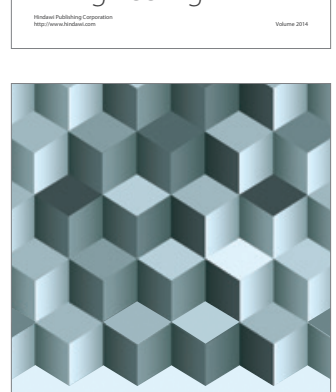

Journal of

Function Spaces
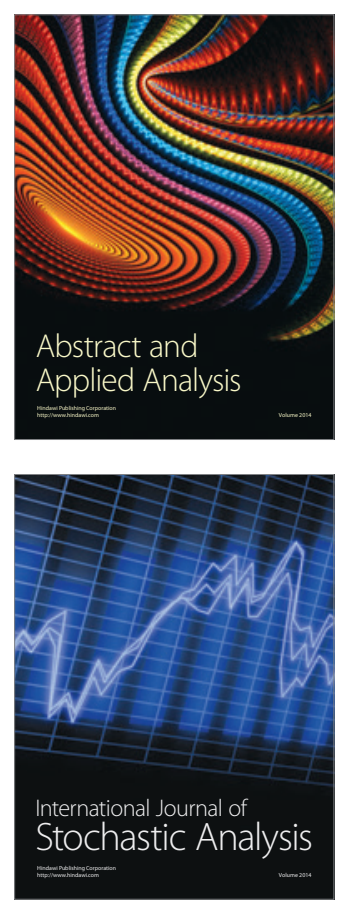

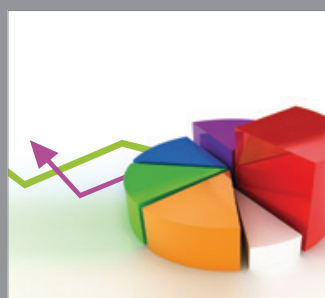

ournal of

Probability and Statistics

Promensencen
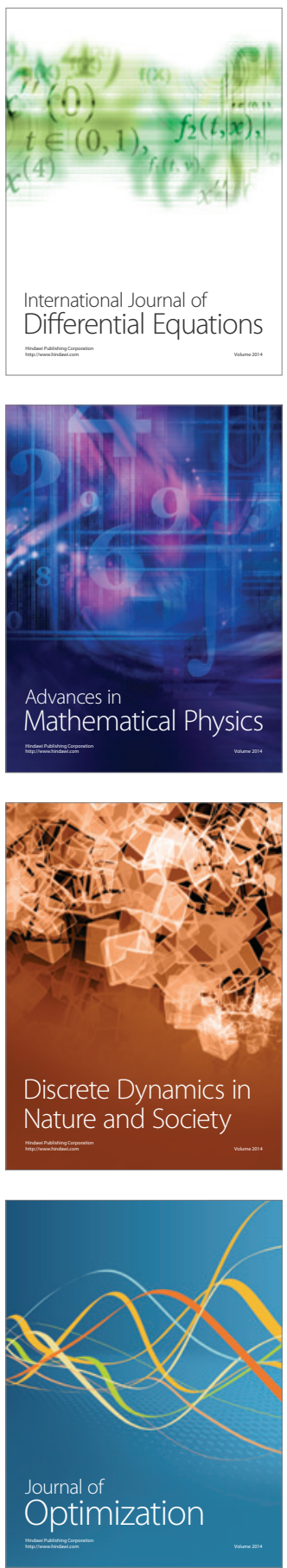